\title{
Thyroid metastatic carcinoma caused by primary lung cancer-a case report
}

\author{
Liang Zhang, Wei Zhao, Jing Chong, Chen Liu, Ning Yu \\ Department of Abdominal Ultrasound, The Affiliated Hospital of Qingdao University, Qingdao, China \\ Correspondence to: Ning Yu. Department of Abdominal Ultrasound, The Affiliated Hospital of Qingdao University, Qingdao 266000, China. \\ Email: 18661808272@163.com.
}

\begin{abstract}
Thyroid metastatic cancer is rare. It may be ignored due to the longer course of the primary disease and the less obvious symptoms. With the popularity of ultrasonography, it can help the early detection of thyroid metastatic cancer. In this case, a 50-year-old woman was diagnosed with thyroid metastasis due to a "decade of lung cancer". The main clinical manifestation of the patient was that although the thyroid area was not enlarged, the texture was stiff. Unlike common thyroid cancer, this patient did not experience hoarseness. The patient underwent ultrasound-guided biopsy of the thyroid area and examined the pathological section. Unlike previous ultrasound manifestations of common thyroid metastatic cancer, the ultrasound results in this case were shown as diffuse, point-like hyperechoic. Ultrasound-guided biopsy of the thyroid site was performed on the patient, and pathological sections were examined. The obtained thyroid pathology section results are shown that metastases of poorly differentiated cancers, tending to adenocarcinoma, considering metastasis in combination with history. The treatment used by this patient was taking thyroxine tablets, but the efficacy was not satisfactory. Early diagnosis of metastatic cancer provides feasible help, and ultrasound-guided puncture biopsy may be used to further diagnose metastatic thyroid cancer if necessary.
\end{abstract}

Keywords: Thyroid metastatic cancer; ultrasonography; diagnostic imaging; case report

Submitted Feb 02, 2020. Accepted for publication May 27, 2020.

doi: 10.21037/apm-20-305

View this article at: http://dx.doi.org/10.21037/apm-20-305

\section{Introduction}

The incidence of thyroid metastatic cancer accounts for only about $3 \%$ of thyroid malignancies (1). Clinically, thyroid metastatic cancer is rare. It may be ignored due to the longer course of the primary disease and the less obvious symptoms. According to reports, common primary tumors of thyroid metastatic cancer are kidney cancer, colorectal cancer, and lung cancer $(2,3)$. So far, only a few articles have reported metastatic thyroid cancer. According to previous reports of ultrasound performance $(4,5)$, thyroid metastatic cancer often appears as a clump of uniform signal, but in this case, the ultrasound shows an unevenly distributed point-like echo. The specific pathogenesis and cause of thyroid metastatic cancer are not clear. Thyroid vessels and lymphatic vessels are abundant, which may be related to non-valvular paravertebral veins. It is generally believed that metastasis to the thyroid indicates that the primary cancer has progressed to advanced stages. We present the following case in accordance with the CARE Reporting Checklist (available at http://dx.doi.org/10.21037/apm-20-305).

\section{Case presentation}

A rare case of lung adenocarcinoma metastasis to the thyroid, one case is now reported: Patient, female, 50 years old, was admitted to our hospital on August 26, 2019 for "ten years after lung cancer surgery, six months after multicourse treatment". This patient had no family history and no abnormal social and psychological history. The patient mainly presented with cough and expectoration and difficulty breathing. According to her medical history: 

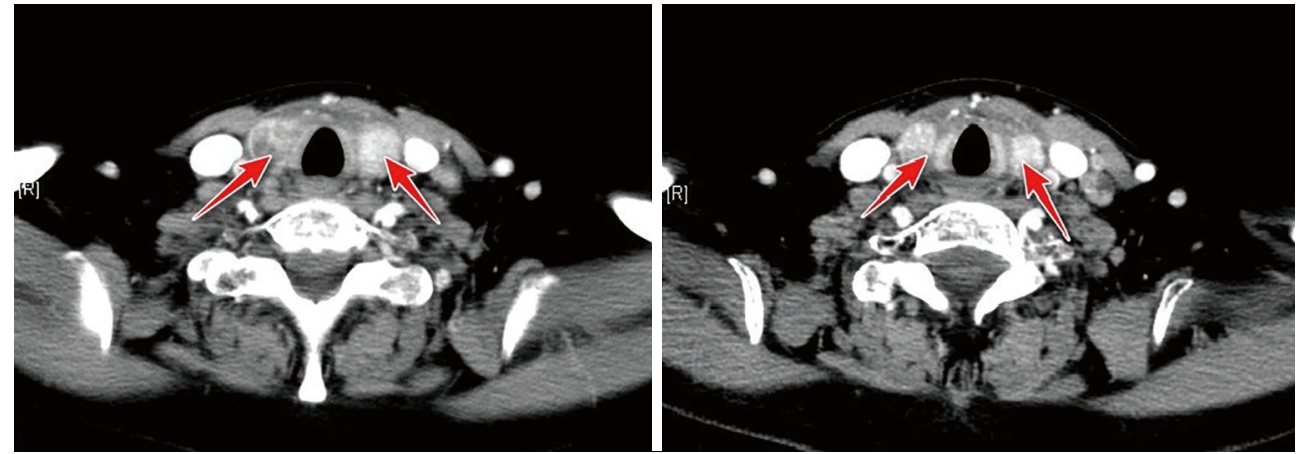

Figure 1 August 26, 2019 neck enhanced CT: bilateral thyroid lobe patchy low density and uneven enhancement. The meaning of arrows is the location of the lesion.
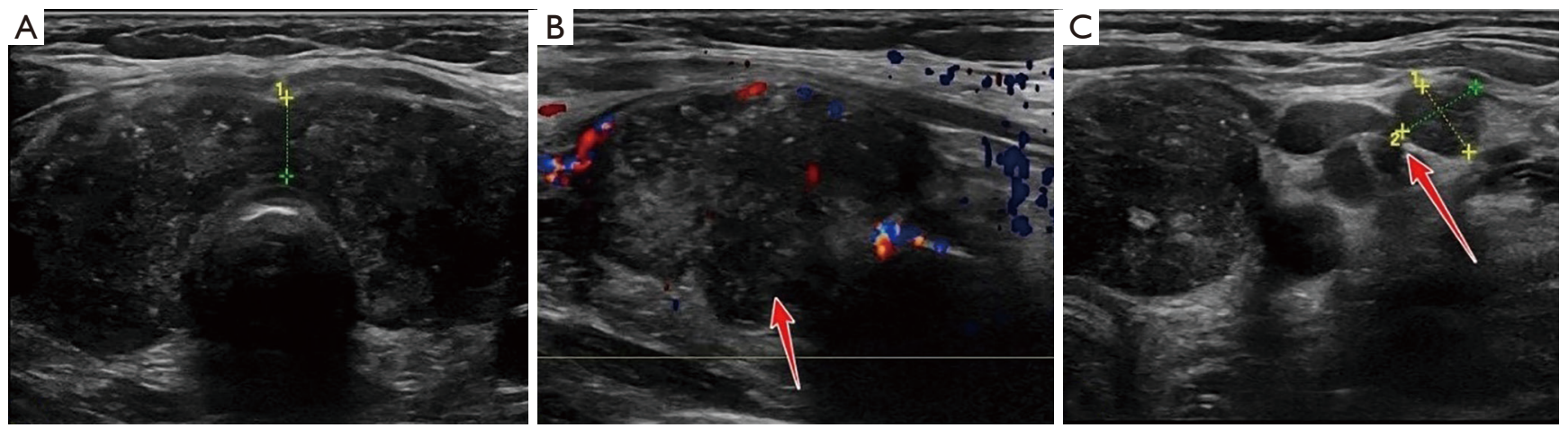

Figure 2 August 26, 2019 Thyroid ultrasound: (A) bilateral thyroid lobe echo reduction, uneven distribution gland; (B) thyroid CDFI showed poor blood supply; (C) the left thyroid swollen lymph nodes. The meaning of arrows is the location of the lesion.

March 5, 2009, left upper lobe resection plus mediastinal lymph node dissection, postoperative pathology: (left upper lobe) centrally differentiated adenocarcinoma. On August 26, 2019, a neck enhanced CT examination was performed (Figure 1): patchy low-density shadows were seen in the double leaves of the thyroid gland, and uneven enhancement was seen, with the right lobe being. Ultrasound examination was performed on August 26, 2019 (Figure 2): bilateral thyroid volume increased, echo within the gland decreased, distribution was uneven, and scattered dot-like high echoes were not detected, and no obvious specific nodules were detected. A large number of enlarged lymph nodes were detected in the right neck VI region, the left neck III and IV regions, the larger was $0.8 \mathrm{~cm} \times$ $0.8 \mathrm{~cm}$, and the portal pulp was unclear. Ultrasound report prompts: thyroid cancer. A Fine Needle Aspiration (FNA) under ultrasound guidance was performed on August 27, 2019, and a Fine Needle Aspiration biopsy was performed on both sides of the thyroid. The results of the pathological examination showed: (FNA biopsy of the right lobe of the thyroid, FNA biopsy of the left lobe of the thyroid) Metastases of poorly differentiated cancers, tending to adenocarcinoma, considering metastasis in combination with history. The patient did not undergo surgery and only took the drug thyroxine for thyroid metastatic cancer, but the efficacy and prognosis were poor. The timeline for the patient's historical development is represented by Figure 3 . Written informed consent was obtained from the patient for publication of this case report and any accompanying images.

\section{Discussion}

With the popularity of ultrasonography, it can help the early detection of thyroid metastatic cancer. According to previous reports, conventional ultrasound detection of thyroid metastatic cancer is mostly manifested as: (I) single or multiple nodular types are common, and multiple 


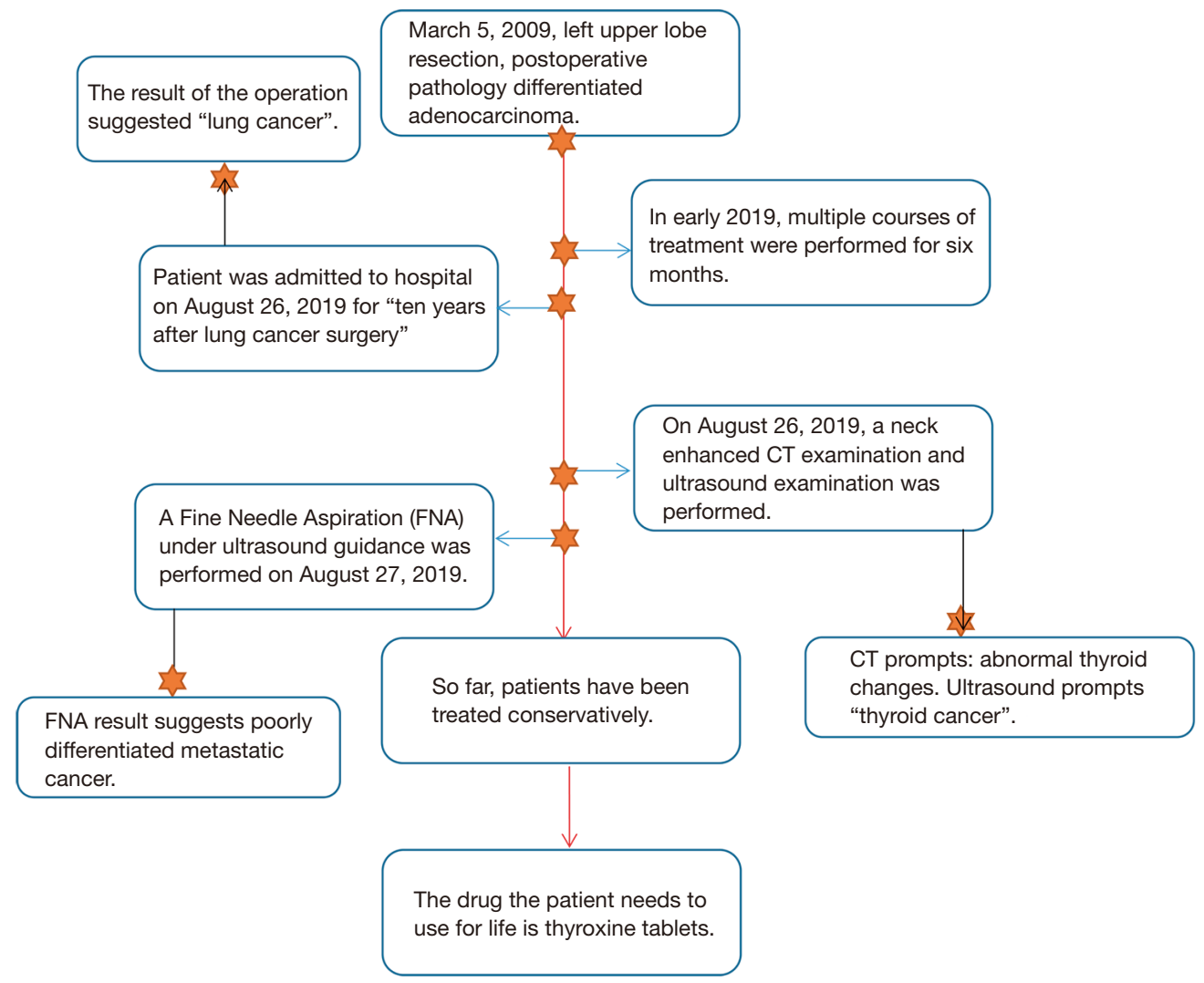

Figure 3 The timeline of historical and current information from this episode of care.

forms are irregular, the border is unclear. (II) Involving bilateral lobes of the thyroid gland. (III) Uneven echo of the glands (6), most of which are hypoechoic, cystic echo can be detected in some cases, and sound transmission is acceptable. The cause of cystic change may be the rapid growth of metastatic cancer, which has insufficient blood supply, and then necrosis liquefies to form cystic change. (IV) CDFI shows abundant blood flow in most cases. (V) There are more cervical lymphadenopathy and calcification. In this case, the patient's ultrasound manifested as diffuse point-like strong echoes scattered in the gland, and no obvious cystic solid mass was seen. This manifestation may be caused by the patient's concurrent Hashimoto's thyroiditis, so the main reason is comorbidity. And in this case, the blood flow signal in the gland was not abundant. Unlike the common thyroid metastatic cancer, it may be diffuse with the lesion, leading to vascular stenosis, which further caused the blood flow signal to be insufficient.

In this case, the ultrasound showed diffuse changes, so it needs to be further distinguished from diffuse thyroid cancer, autoimmune thyroid disease, and lymphoma. (I) Diffuse thyroid cancer has no obvious masses and nodules. Gland echoes generally increase. CDFI shows abundant blood flow in the glands, and micro-calcifications of point hyperechoicity can be seen. Ultrasound detection of grit in the gland can be the basis for the diagnosis of thyroid cancer (7). (II) Ultrasound manifestations of autoimmune thyroid disease are diffuse hypoechoic, whose typical manifestation is "grid-like", and CDFI shows abundant blood flow signals. (III) Patients with lymphoma generally have a history of Hashimoto's thyroiditis, and the typical surface is a rapid increase in painlessness of the neck mass, a large lesion, a clear border, and the thyroid capsule is not damaged, and the echo within the lesion is reduced. The rear echo is enhanced.

Clinically, the gold standard for the diagnosis of thyroid metastatic cancer is pathological examination. CT diagnosis of thyroid metastatic cancer has no specific manifestations, but if combined with the history of primary tumors, the age is $>45$ years, and CT indicates a significant space, or thick 
Wall cystic changes should take into account metastatic thyroid cancer. And immunohistochemistry is more likely to be used as a differential diagnosis method for primary tumors and thyroid metastatic cancers. Tg, TTF-1, calcitonin, etc. are often positively expressed in thyroid cancers, and the markers expressed in thyroid metastatic cancers are often the same as those in the primary carcinoma. So far, symptomatic treatment is generally used for patients with thyroid metastatic cancer. The occurrence of metastases usually means that the primary disease is severe and serious. Surgical resection for the primary tumor is the best method. However, due to the special location of the metastasis, if it is not the symptoms of compression of the trachea by the metastasis, surgical resection is generally not recommended. In this case, the patient originally intended to undergo surgical treatment. But she listened to the doctor's suggestion and combined with her own situation, the patient chose thyroxine drugs to control the progress of the disease. The thyroxine drug selected for the treatment of thyroid metastatic cancer was taken for life. After contacting the patient by phone, the patient reported that her physical condition was the same as before and did not improve. The patient reported that the thyroid gland did not touch the enlargement, but the symptoms of dyspnea were not significantly relieved. Judging from the patient's symptoms, the patient's compliance with the intervention is poor, which may be related to her own physical condition. The advantage of this case lies in the rare ultrasound manifestations of this case, which can further enrich the types of clinicians' diagnosis of the disease. However, the limitation of this case is that the patient did not undergo surgical treatment due to her own condition and could not evaluate the prognosis of the disease. The principle of the patient's treatment was to control the disease without threatening her life. Due to the poor condition of the patient, drug intervention was adopted. In the next course of drug use, patients and their families expect to be effectively controlled and her symptoms improved. With a deeper understanding of thyroid metastatic cancer and an increase in the detection rate of thyroid metastatic cancer, clinicians should increase their awareness of the possibility of thyroid metastatic cancer.

\section{Conclusions}

Thyroid metastatic cancer is a clinically rare disease with a low incidence and a high rate of misdiagnosis. It is difficult to detect. Therefore, the diagnosis rate of thyroid metastatic cancer needs to be increased. Early diagnosis of metastatic cancer provides feasible help, and ultrasound-guided puncture biopsy may be used to further diagnose metastatic thyroid cancer if necessary.

\section{Acknowledgments}

We are thankful to Dr. Jiang from the department of Pathology, the Affiliated Hospital of Qingdao University, who provided the confirmation of the histologic diagnosis of the patient.

Funding: None.

\section{Footnote}

Reporting Checklist: The authors have completed the CARE Reporting Checklist. Available at http://dx.doi. org/10.21037/apm-20-305

Conflicts of Interest: All authors have completed the ICMJE uniform disclosure form (available at http://dx.doi. org/10.21037/apm-20-305). The authors have no conflicts of interest to declare.

Ethical Statement: The authors are accountable for all aspects of the work in ensuring that questions related to the accuracy or integrity of any part of the work are appropriately investigated and resolved. All procedures performed in studies involving human participants were in accordance with the ethical standards of the institutional and/or national research committee(s) and with the Helsinki Declaration (as revised in 2013). Published reports and pictures have been obtained from patients with informed consent. And we guarantee the true validity of the case.

Open Access Statement: This is an Open Access article distributed in accordance with the Creative Commons Attribution-NonCommercial-NoDerivs 4.0 International License (CC BY-NC-ND 4.0), which permits the noncommercial replication and distribution of the article with the strict proviso that no changes or edits are made and the original work is properly cited (including links to both the formal publication through the relevant DOI and the license). See: https://creativecommons.org/licenses/by-nc-nd/4.0/.

\section{References}

1. Nixon IJ, Coca-Pelaz A, Kaleva AI, et al. Metastasis to 
the Thyroid Gland: A Critical Review. Ann Surg Oncol 2017;24:1533-9.

2. Russell JO, Yan K, Burkey B, et al. Nonthyroid metastasis to the thyroid gland: case series and review with observations by primary pathology. Otolaryngol Head Neck Surg 2016;155:961-8.

3. Cai C, Shen H, Liu W, et al. Rare metastasis of nasopharyngeal carcinoma to the thyroid gland with subsequent metastatic abdominal lymph nodes: A case report and literature review. Medicine (Baltimore) 2017;96:e8373.

4. Andrioli M, Persani L. Elastographic presentation of

Cite this article as: Zhang L, Zhao W, Chong J, Liu C, Yu N. Thyroid metastatic carcinoma caused by primary lung cancera case report. Ann Palliat Med 2021;10(6):7046-7050. doi: 10.21037/apm-20-305 synchronous renal cell carcinoma metastasis to the thyroid gland. Endocrine 2014;47:336-7.

5. Zhang L, Liu Y, Li X, et al. Metastases to the thyroid gland: a report of 32 cases in PUMCH. Medicine (Baltimore) 2017;96:e7927.

6. Choi SH, Baek JH, Ha EJ, et al. Diagnosis of metastasis to the thyroid gland: comparison of core-needle biopsy and fine-needle aspiration. Otolaryngol Head Neck Surg 2016;154:618-25.

7. Richard J. Rosai and Ackerman's Surgical Pathology: Ninth Edition. Pathology, 2004;36. 